\section{Mohammad Ayed} Aloufi $^{1}$

Article info: Received 31.01.2020 Accepted 16.04.2020

UDC - 05.311.6:.614.253.5 DOI - 10.24874/IJQR14.03-01

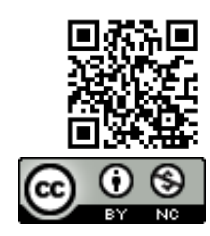

\title{
EFFECT OF CLINICAL DECISION SUPPORT SYSTEMS ON QUALITY OF CARE BY NURSES
}

\begin{abstract}
The aim of this review was to examine to what extent the need for nursing specific Clinical Decision Support Systems has been realised in terms of progress in research on this topic, areas of such researches and the findings of these researches. The topic title itself was used as the search term in Google Scholar for different time frames and only papers which have nursing CDSS in their titles were selected. This process yielded 32 papers, which were listed and described briefly. From the listed and described 32 papers, some trends on types of papers and assessment results were tabulated in terms of number of papers in each category and the pertinent references. Three tables were obtained in this manner. The maximum number of papers dealt with evaluation or effects of nursing CDSS. There were nine reviews on the topic spread over time. Seven papers had factors or barriers as their topics. Notably, only one paper dealt with use of mobile devices in nursing CDSS in spite of the fact that use of smart phones is rapidly increasing in every part of life. While 14 papers assessed CDSS positively, seven assessed it negatively. Another seven were neutral in assessment or there was no clear statement on this. Three papers dealt with scope for nursing CDSS, which should prompt more research on this. Reviews were mostly used. There were only two papers which used high quality RCT, The findings of the review are discussed using appropriate arguments and scope for future research has been indicated at each point and listed at the end. Some limitations of this review have also been listed.
\end{abstract}

Keywords: Decision Support System; Quality of Care; Nurses

\section{Introduction}

Clinical decision support system brings together clinical information about patients into an IT system. Such systems are used by health practitioners and others to aid their decisions. For the Stage 2 EHR Incentives Programmes, the Office of National Coordinator, USA defined CDSS as, "HIT functionality that builds upon the foundation of an EHR to provide persons involved in care processes with general and person-specific information, intelligently filtered and organized, at appropriate times, to enhance health and health care." (Bresnick, 2017).

In the site of HealthIT, ONC gives another definition of CDSS, "Clinical decision support (CDS) provides clinicians, staff, patients or other individuals with knowledge and person-specific information, intelligently

\footnotetext{
${ }^{1}$ Corresponding author: Mohammad Ayed Aloufi Email: mohdaloufi@hotmail.com
} 
filtered or presented at appropriate times, to enhance health and health care." (ONC, 2019).

The definition given in $\mathrm{CDC}$ is, "Clinical decision support systems (CDSS) are computer-based programs that analyse data within EHRs to provide prompts and reminders to assist health care providers in implementing evidence-based clinical guidelines at the point of care." (CDC, 2018).

According to AHRQ, "Clinical decision support (CDS) provides timely information, usually at the point of care, to help inform decisions about a patient's care. CDS tools and systems help clinical teams by taking over some routine tasks, warning of potential problems, or providing suggestions for the clinical team and patient to consider." (AHRQ, 2019).

All the above definitions focus on computerised healthcare in which part of the decision making is shifted to the knowledgebase in the computer (via internet) or decisions of healthcare professionals are facilitated with supporting data. The latter types do not interfere with the basic role of the healthcare professionals like clinicians or nurses. But if decisions are taken over by machines, the role of healthcare professionals in quality of care may diminish.

The above reservations may be particularly true in the case of nurses. Nurses are the immediate contact point for patients. Quality of care starts from there. Many nursing researchers have highlighted the important role of nurses beside the patients in determining current status of disease, effect of interventions and need to change any element of care. Clinicians depend on nurses, who are with the patients for 24 hours, for such assessment and need to change any of the care components.

Therefore, a mistake at this point can lead to serious medical errors eve to the extent of patient mortality. Errors in personal judgement of these aspects by nurses need to be minimised to minimise medical errors.
This means, personal judgement by nurses is highly prone to error particularly when they are under high levels of work pressures and shift problems and tensions due to work-life imbalances. Even if these problems are reduced by the management adopting suitable strategies, some scope for such errors still remain. It is here that some guidance, warning or alert when the nurse is likely to make errors is available in the system. It will be even more useful if the system can protect the nurses from the possibility of any errors by ensuring evidence-based best practices and standards of quality care.

Thus prevention is better than cure. The subject of nursing-specific CDSS (distinct from clinician or hospital specific) assumes importance here. It is important that any CDSS applicable to any nursing context should be specific to nursing needs and not generally applicable, as was stressed by Arnaert et al. (2017) in one of the reviewed papers below.

Therefore, in this review paper, we examine to what extent nursing CDSS research has progressed and in what areas such researches have been done and what were the findings of these researches.

\section{Methodology}

The papers related to the topic were searched by directly using the topic of this review itself as the search term in Google Scholar for both older and recent papers. Only the papers which had nursing CDSS in their titles were selected. Only English language papers were selected.

Even abstracts were included if they contained essential points. If full texts of chapters in books were available, they were also included.

The search using the two types of time frame yielded 32 papers. These are discussed in the following sections. 


\section{Result}

\subsection{List of selected paper and brief descriptions}

Anderson and Wilson (2008) systematically reviewed CDSS in nursing. The authors sought answers for the questions on the progress made in the development of CDSS and its use in nursing; research methods and theoretical models applied by researchers on this topic and how much of evidence-adaptive CDSS is applicable specifically in nursing.

Enhancement of nurses' capacity for decision making through CDSS was explored by Thompson et al. (2013) using systematic review. Mixed results have been reported on this aspect in different papers. Recently, positive effects of CDSS on nursing care has been demonstrated in specific situations. As per the observation of the authors, if CDSS can be linked to mobile devices like smartphones in the hands of nurses, it could produce more positive results.

A systematic review employing Cochrane and AMSTAR standards by Jaspers et al. (2011) found high quality evidence only in the case of very few papers. Patient outcome benefits were found only in the case of drug ordering and preventive care reminders. The study included nurses also.

The effect of a nurse expert system UNIS was found to be similar to the knowledge level of expert nurses in caring elderly incontinent patients in nursing homes, according to the results obtained by Petrucci, et al. (1991) from a pair of trials.

The results of an integrated review on the effect of CDSS on registered nurses in acute care settings was reported by Dunn Lopez, et al. (2017). Clinical support systems specifically meant for bedside nurses showed positive effects on outcomes and hold promise for improving care quality. Most of the CDS were used by nurses to support diagnostic decision-making, adherence to guidelines and management of medication and situational awareness. In a few studies, patient outcomes improved significantly when CDS was used by nurses. There was no negative effect of CDS on process, usability or patient outcomes.

A qualitative review of works on nurses' use of CDSS by Piscotty and Kalisch (2014) identified factors related to nurses, patients, technology and design and organisation, affecting their use of CDSS. The CDSS were not designed primarily for their use. So, these systems may not have the expected effects on patient outcomes or quality of care.

Nursing processes consisting of state of the art, standardised classifications of care, are called Advanced Nursing Process (ANP). Valid assessments, nursing diagnosis, interventions and nursing-sensitive patient outcomes are part of ANP. CDSS can facilitate use of ANP by nurses. The study by Müller-Staub et al. (2016) was aimed at developing a validated nursing CDSS. A 28criteria system was developed, pilot-tested with 29 nurses and based on the results, the number of criteria were reduced to 25 . Expert consensus and content validity were obtained for 14 criteria. The need for more preresearch based predefined nursing diagnosis criteria and linking them with evidence-based interventions, diagnosis and patient outcomes were suggested as further improvement on the nurse-centric CDSS model. The authors provide a list of previous attempts, as reproduced in figure 1 .

All the seven systems were developed, clinically applied and psychometrically tested in specific national contexts. Only one of them, the Quality of Diagnoses, Interventions, and Outcomes (Q-DIO), was an SNL-based CDSS. It is aimed to measure the associations between assessments, diagnoses, interventions and outcomes in a coherent and accurate way. It has 29 items, is a criterion-referenced instrument, available in seven languages and has been psychometrically tested in many national settings.

Based on the findings, the Q-DIO can be used as the basis for developments in EHR. 
D-Catch has 10 items for measuring accuracy of admission data, nursing diagnosis and interventions, progress notes, outcome evaluations and even legibility of their notes. However, none of the seven was usable for measuring the accuracy of ANP documentation in EHRs. Multicentre study was done only in the case of Q-DIO. Thus, the development of the CDSS for ANP in this paper is justified.

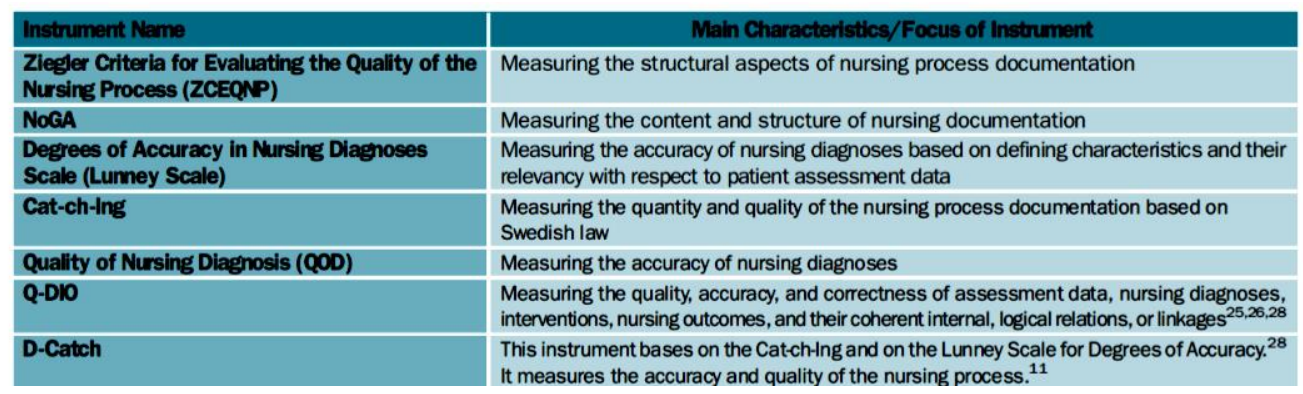

Figure 1. Nursing decision support systems already reported in Literature

(Müller-Staub et al., 2016)

No effect was found by Fossum et al. (2011) on the Risk Assessment Pressure Scale (RAPS) for PU risk screening and the Mini Nutritional Assessment (MNA®) scale for screening nutritional status when using a CDSS-integrated EHR by nurses. However, there was a decrease in the frequency of malnutritioned patients.

Based on a systematic review, Randell et al. (2007) concluded that CDSS by itself may not lead to improved outcomes related to either nursing performance or patient outcomes. Research works on both nurses using CDSS versus nurses not using CDSS and nurses using CDSS versus other healthcare staff not using CDSS were compared.

Results of participant observation by Dowding, et al. (2009) showed that nurses used CDSS for recording information, monitoring of patients' progress and confirming the already made decisions. The personal experience of nurses in decision making and technology use determined the extent and method of their using CDSS. The extent of their overriding the CDSS recommendations was also a factor. Their ability for technology adoption also affected use of CDSS.
Organisational factors for implementation of a nursing CDSS in NHS hospitals UK were evaluated by Randell and Dowding (2010). System characteristics, training, clinician engagement and IT infrastructure, resources were identified as the main organisational factors from stakeholder interviews, participant observations and other qualitative methods in four case sites.

Lack of structured and coded diagnosis documentation system and outdated medication system in the EHR stood in the way of achieving the full benefits of CDSS. These problems led to false alerts due to which the trust on the system was reduced. On the other hand, timely reminders of otherwise ignored matters, automatic calculations helping determination of correct medication rates, safety checks, summarised medical reviews were beneficial as was reported from a Finnish study by (Koskela et al., 2015).

Successful implementation of decision support adjusted for patient-preferences was reported by Ruland (1999), based on quasiexperimental research. Information about patient preferences changed. The care priorities of nurses could be adjusted to patient preferences, so that patient performance and satisfaction were better. 
Use of handheld devices for decision support by nurses improved their performance in relationship with patient-preferred interventions and patient outcomes. This was a quasi-experimental study by Ruland (2002) similar to the paper described above.

From a time-series based design, Lyerla et al. (2010) obtained increased adherence to guidelines by nurses when nursing CDSS was integrated into a patient's electronic flow sheet in the case of patients using mechanical ventilation.

A nursing CDSS system for placing mechanically ventilated patients in a semirecumbent position when there is no contraindications was developed and implemented by Lyerla (2008). A review of literature on related aspects, expert guidelines and CDSS provided the basis of the CDSS developed by the author. The CDSS acted as a reminder for nurses to adhere to the guidelines.

From a survey of nurse anaesthetists, Hsiao et al. (2013) noted dependence of nurse accepting the CDSS on pain management system on their perceived information quality, computer self-efficacy and organisational structure, the latter two affecting their perceived usefulness. These variables had relationships with perceived ease also.

Lee (2013) reviewed the system features of CDSS for nursing practices. It was observed that in the features represented the characteristics of each stage from patient assessment to the outcome evaluation. All reviewed papers provided sequential plans for patient assessment and care plans in the decision support. A few of them also had problem identification. An implementation stage was included in some of them and some others included outcome evaluation. Results demonstrated the sequence of decision support steps as: initial patient assessment, problem identification, care plan and outcome evaluation. CDSS covering the entire range of scope effectively were helpful for clinical decision making by nurses. Such sequential organisation of the system features facilitated getting a comprehensive knowledge of CDSS particularly focused on nursing practices.

Hao et al. (2013) developed a decision support system for nursing process (NPDSS) in the urology ward. It was based on three clinical pathways: benign prostatic hypertrophy, inguinal hernia, and urinary tract stone. NPDSS was developed for six major nursing diagnoses: acute pain, impaired urinary elimination, impaired skin integrity, anxiety, infection risk, and risk of falling. Consensus of decision support rules of NPDSS was reached using a Delphi method in which nine expert nurses participated. Six nurses were used for evaluation of the system developed.

A focus group was employed by Choi et al. (2011), in which 37 Korean nurses participated to identify issues and contents required for a CDSS for nursing-sensitive outcomes on patient safety in their critical care clinical practice settings. Issues of threats to patient safety and nursing-sensitive outcomes were identified. The types and contents of CDSS the nurses preferred were notifications, alerts, reminders and warning systems; guidelines on point-of-care and reference sources for information/guidelines. Nurses strongly believed that CDSS could help them to improve nursing outcomes by facilitating application of standardized nursing care.

A study to understand the steps of knowledge definition, collection, and representation in the nursing process was undertaken by Kim et al. (2006). These were meant to be used as a design a data warehouse to develop a nursing process CDSS.

Dowding, et al. (2009) examined how experience of nurses determines their use of CDSS. Interviews and observations from two case studies were used as the data. Integration of knowledge obtainable from CDSS and effect of experience on it emerged as the themes.

In Iran, Agharezaei et al. (2014) implemented a CDSS in hospital. The system was capable 
of identifying patients at the risk of pulmonary embolism (PE) and deep vein thrombosis (DVT). It was also capable of producing reminders for prophylactic action against these diseases. Using semi-structured interviews, the attitude of doctors and nurses towards this system was assessed. Both physicians were positive about the beneficial effects of the intended functions and thought that it would improve their own performances as well.

Borum (2018) identified 13 barriers to adoption of CDSS by nurses through a systematic literature review. Having right information, up-to-date evidence-base for practice guidelines, precise clinical pathways, current clinical algorithms were more common barriers out of them. Reliable CDSS is important as nurses increasingly rely on technology. Elimination of these barriers will improve their acceptance and use of CDSS.

In another systematic review, Van de Velde et al. (2018) concluded that to be more effective, CDSS should generate advices were automatically displayed on-screen and when they were more patient-specific. Improvement in adherence was possible if CDSS interventions were combined with other strategies. Using CDSS directly on patient also showed better adherence. Economic outcomes and patient satisfaction were researched rarely. Certainty of the evidence obtained was in the range of low to moderate for all these results.

The feasibility of a protocol to simultaneously compare different CDS formats was examined by Keenan, et al. (2017) A simulated environment was used for evaluating the effects of numeracy and graph literacy in the adoption of best practices and time planning of care by registered nurses (RN). A factorial experiment involving 60 RNs was done with one control and three CDS groups. The exercise enabled the identification of 14 best practices for two patients. These were sent in the form of evidence-based suggestions to the three experimental CDS groups. In comparison with control group, the CDS groups recorded a higher rate for adoption of best practices in all shifts and reduced care planning time in later shifts as compared to the control group. There was association of higher numeracy and graph literacy with shorter care planning times for two CDS groups. However, the three CDS groups did not differ significantly with respect to adoption rate and care planning time. Thus, the feasibility of the protocol was established. CDS clearly showed improvement in the efficiency and effectiveness of care planning decisions. But the optimal format might be determined by the characteristics of each registered nurse.

Evidence-based education only temporarily increased the rate of nurse documentation of EBP. Nurses might not have documented all their EBP during their duty. A longitudinal cluster-randomised design with self-reported 200 patient records as data elements was used in a study by Susan Cortez (2016) spread over 11 weeks to report these findings.

In a book chapter, Arnaert et al. (2017) observed that CDSS has been used extensively for medicine, but not for nursing practices. Some prototypes, which already exist, are not tuned to nursing practices. Even at advanced levels of nursing practices similar to clinicians, the scope of CDSS has not been examined well. The nurses are expected to possess very large amount of knowledge. This makes their cognitive load very high. CDSS will help to reduce this cognitive load. It may be necessary to identify and remove any obstacles for developing successful nurse-specific CDSS.

The CDSS is engineered to facilitate clinical decision- making processes by analysing the characteristics of individual patients to generate patient-specific recommendations. The results are presented in a form actionable at end-user levels consisting of an alert, reminder, order set or drug-dose calculation. It may also be in the form of a care summary dashboard to display patient care quality in terms of established quality indicators. However, in majority of cases (49-96\%) such 
alerts and advices from the CDSS are ignored and different actions are taken. Ignoring the alerts and substituting with own judgement may cause medical errors and other adverse events like patient mortality. The authors examined the effects of "hard stop" documentation, which prevents further action unless the prescribed procedure is completed and reported. However, end-user experience of "hard stops" are not positive. Introduction of best practices CDSS without the need for "hard stops" may only be the solution (Gold et al., 2018).

If the perceptions of RNs on using a CDSS in drug monitoring are known, it may be possible to develop safer practices in drug management. The results of interviews conducted by Johansson-Pajala et al. (2017) showed that RNs supported the idea, but were concerned about timeliness, standardisation of clinical work. Obtained knowledge, evidence and separation of responsibilities between clinicians and nurses were also important.

From the results of an experimental study, Geurts, et al. (2017) observed that nurseguided CDSS improved compliance and increased the standardised use of the online registration system.

There is good potential for CDSS to improve patient outcomes by providing evidencebased guidance to frontline nurses in the course of their workflow. Glasofer (2016) explored the effect of a CDSS in promoting nurse-directed catheter removal protocols on their rates. Nurses were cautious in complying with CDSS guidance due to their perceptions of professional values in which autonomy, accountability, and high-quality care are more important. They also wanted to avoid conflict in professional relationships and achieve easy workflows. About half of the interviewed nurses were not comfortable to remove the catheter without minimally consulting with the provider or wanted an order from the provider. This discomfort was due to the strong belief that nurses are unable to function without being ordered for every action even if they were aware of standing protocol. They also feared that if the catheter had to be re-inserted, the patient or physician might become angry with them.

\subsection{Trends of Topic}

Table 1 provides the general trend of topics identified in the selected papers. Nine papers dealt with design and development of nursing CDSS. However, 12 papers evaluated or measured the effects of CDSS in different context settings. There were 7 studies which dealt with factors or barriers to implementation of nursing CDSS in various settings. Although use of mobile phones is rapidly increasing and $m$-health has become an actively researched area, there was only one paper which considered the scope of using mobile devices for increasing the efficiency and effectiveness of nursing CDSS.

Table 1. General trend of topics in the selected papers

\begin{tabular}{|l|c|c|}
\hline Type of paper & $\begin{array}{l}\text { No of } \\
\text { papers }\end{array}$ & References \\
\hline $\begin{array}{l}\text { Reviews/Systematic } \\
\text { reviews }\end{array}$ & 9 & $\begin{array}{c}1,2,3,5,6,9, \\
18,24,25\end{array}$ \\
\hline CDSS development & 3 & $7,19,21$, \\
\hline $\begin{array}{l}\text { CDSS } \\
\text { evaluation/effect }\end{array}$ & 12 & $\begin{array}{c}4,8,10,12, \\
13,15,16,23, \\
26,27,29,31\end{array}$ \\
\hline $\begin{array}{l}\text { CDSS } \\
\text { factors/barrier }\end{array}$ & 7 & $\begin{array}{c}11,17,20,22, \\
28,30,32\end{array}$ \\
\hline $\begin{array}{l}\text { CDSS handheld } \\
\text { devices }\end{array}$ & 1 & 14 \\
\hline
\end{tabular}

The methodological approach used in the 32 selected papers have been tabulated in Table 2.

About one-third of the papers (11 in all) were reviews, of which about $69 \%$ (7 out of 11 ) were systematic reviews. Three papers used interviews. More than one method were used in three papers. Other methods of research were almost evenly distributed among the papers. 
Table 2. Research methods used.

\begin{tabular}{|l|c|c|}
\hline Research method & $\begin{array}{c}\text { No of } \\
\text { papers }\end{array}$ & \\
\hline Systematic review & 7 & $1,2,3,5,9,24,25$ \\
\hline Qualitative review & 3 & $6,28,29$ \\
\hline Selective review & 1 & 18 \\
\hline Trials & 1 & 4 \\
\hline More than one method & 3 & $7,11,22$ \\
\hline Screening & 1 & 8 \\
\hline Participant observation & 1 & 10 \\
\hline Focus group & 2 & 12,20 \\
\hline Quasi-experimental & 2 & 13,14 \\
\hline Time series design & 1 & 15 \\
\hline Design, testing and implementation & 2 & 16,19 \\
\hline Survey & 1 & 17 \\
\hline Data warehouse design & 1 & 21 \\
\hline Interviews & 3 & $23,30,32$ \\
\hline Factorial experiment & 1 & 26 \\
\hline Longitudinal cluster-randomised design & 1 & 27 \\
\hline Experimental study & 1 & 31 \\
\hline
\end{tabular}

It is also possible to categorise the selected papers based on whether they reported positive or negative impressions about nursing CDSS. The results of such an analysis is presented in Table 3 .

Table 3. Positive and negative assessments of nursing CDSS in selected papers

\begin{tabular}{|l|c|c|}
\hline $\begin{array}{l}\text { Assessment } \\
\text { type }\end{array}$ & $\begin{array}{l}\text { No of } \\
\text { papers }\end{array}$ & References \\
\hline Positive & 14 & $\begin{array}{c}5,13,14,15,16, \\
18,19,20,21,22, \\
23,26,30,31\end{array}$ \\
\hline Negative & 7 & $\begin{array}{c}6,9,12,24,25,27, \\
29\end{array}$ \\
\hline Neutral & 7 & $\begin{array}{c}1,4,7,8,10,11, \\
17,\end{array}$ \\
\hline Mixed & 1 & 2 \\
\hline Scope & 3 & $3,28,32$ \\
\hline
\end{tabular}

Out of 32 papers reviewed, 14 papers assessed nursing CDSS positively on various counts depending on the nature of problem being studied. Seven papers each assessed nursing CDSS either negatively or neither positively or negatively in a clear manner. Mixed types of results were reported by one paper, although this had been a major point discussed in many review papers. The potential or scope of CDSS were examined in three different ways in as many papers.

\section{Discussion}

It is possible to consider the 32 selected papers as stand alone works if they are assessed minutely. Each paper considered a specific context. Each of them applied specific methodology which the researchers thought appropriate, but sometimes questionable on the sample size and approaches used in relation to the research aims. The results obtained were interpreted in their own ways, although alternate explanations might be possible in some cases. Conclusions in some papers were beyond the scope of their investigation.

In spite of the above problems with individual papers, some common trends were obtained as was given in the tables above. Table 1 was related to the type of paper. The categorisation used in the table was somewhat arbitrary. Sometimes, the findings cannot be clearly categorised into one or the other type. In such cases, the major finding or conclusion was used for categorisation. Overlap between categories is a definite possibility. 
From Table 1, reviews were maximum. That means there had been sufficient number of papers on the topic before the year of review. However, in many of these reviews, a large number of papers were screened, and their quality assessed very strictly.

This process resulted in exclusion of a large number of papers as poor quality. The so called poor quality papers would have been some preliminary study meant to just understand the possibility of using nursing CDSS in a specific context. Unless the aim is related with how the work was done, the categorisation into poor quality may not be justifiable. To expect a randomised replicated trial in an exploratory study may be too much. In these respects, methods like those of Cochrane may need to be reviewed.

When a large number of studies on CDSS was assessed as poor quality, it highlights the need to develop more CDSS. Only three papers made any serious effort here. Of particular interest is the paper of Müller-Staub et al. (2016), who studied all angles related to the issue of developing new CDSS quite exhaustively.

The fact that so many reviews were done highlights the availability of many already developed CDSS. It is only natural that most of the selected works dealt with evaluation or finding the effects of nursing CDSS in various situations. The range of contexts or situations covered by these 12 papers was so wide that almost all critical aspects of care quality were covered and only very few were left for further research. Some the uncovered areas in the selected papers might have been obtained if the literature search was expanded to more than five pages of Scholar. This can always be thought as a limitation of this work.

When any new system needs to be implemented, barriers are certain. Therefore identification of barriers is extremely important.

Considering the variety of contexts, only seven papers dealt with this aspect and this appers to be too insufficient. There is need for more research in the areas of barriers.
Sometimes, barriers are not clearly visible. Some papers identified barriers by deducing from the results rather than directly. The extent to which each barrier needs to be assessed to determine the priorities of tackling them. But the papers did not give too much attention to this aspect.

A wide variety of research methods were used in the selected papers. The preferred method was review of literature, systematic or otherwise. However, unless a large number of works are available reviews may not yield adequately generalisable trends as this review itself shows. The appropriateness of the method selected in some papers may be subject to question. A systematic review of research methods used may be able to say about the appropriateness.

Usually, randomised control trials are considered to be the highest quality of evidence. However, such methods were used only in two works. Thee need for more RCT is evident here.

The matter of immediate interest is how many of the reviewed papers reported positive assessment of nursing CDSS. This will indicate how well the idea of nursing CDSS has been received. As shown in Table 2, 14 out of 32 papers (44\%) displayed positive assessment of CDSS. Although this is a good number, it is short of even $50 \%$ of the selected papers. It also means, the rest of $56 \%$ did not quite assess nursing CDSS as positive. The positive assessment in these papers were due to some factors or aspects of CDSS producing positive results. Some papers reported certain benefits due to CDSS. It could be argued that more papers might exist which may have escaped inclusion due to the search strategy restrictions of this review.

When the number of papers assessing negatively and non-negatively is considered, only seven papers $(22 \%)$ were distinctively negative. The balance 25 papers $(78 \%)$ did not assess nursing CSSS as negative. Viewed from this angle, $78 \%$ not assessing CDSS negatively, is certainly welcome. In the given contexts, CDSS was either inferior to the 
current systems or CDSS did not produce favourable or expected results. The expected results are in terms of increase or decrease of some variables, some specific benefits not in line with the expectations or even some adverse effects. Sometimes such effects could not be directly attributed to CDSS but inferred indirectly from certain trends of results.

Another seven papers did not assess CDSS either positively or negatively. This was due to the difference in their focus. The direction of assessment was determined from implicit findings derived from the observations. One paper by Keenan et al. (2017) dealt with comparison of different CDSS frameworks.

Another important point is about the scope of nursing CDSS. As was pointed out by Arnaert et al. (2017), the usefulness of CDSS in medicine is well-known and hence widely used. But nurse-specific CDSS has not received much attention. This is the most important reason for development of nursing CDSS now and in future. Only three papers showed the scope of developing and potential benefits possible through nursing CDSS. Here, the need for more research is strongly indicated.

\section{Conclusion}

Although a large number of papers give the message that nursing CDSS is highly beneficial in many ways, certain aspects in the development, evaluation and implementation still need fine tuning through more research. There is need to develop standards, evidence-based practices and guidelines for nursing CDSS. The methodology used for development and evaluation need to be more scientifically valid. A significant challenge in all these matters is that, CDSS to be developed and used might be different for different intervention contexts like emergency department, oncology, surgery and management of chronic diseases.
Only a small number of areas out of these have been covered in the current research reports. Researches on other areas are yet to begin. However, in summary, it can be said that whatever research has been done on nursing CDSS have highlighted the importance of having them considering the great scope and potential for their utility.

\subsection{Limitations}

The use of topic title itself as search term is not the standard practice. In defence, it is much easier to find papers with such a strategy rather than using many search terms with words broken from the title or other related words, such strategies using many databases yields thousands of papers and filtering steps results in very few papers. Therefore, the search method adopted here is superior.

The second limitation is restricting the pages to five in the search engine. Important papers beyond the 5th page may escape selection. But this risk always exists even if 10 pages are selected. The five pages was chosen to increase search efficiency. A general Google search yielded either some of the already selected papers and did not increase the search result appreciably.

The third limitation may be restricting the selection to only those papers which were specifically titled containing the words nursing CDSS. A test by selecting some other papers showed that they did not mention nursing CDSS anywhere in the paper or it was not any significant part of the work. This selection restriction worked well in the desired manner.

\subsection{Scope for Future Research}

Mention about the need for further research had been done in the discussions above. A few more important ones are listed below:

a) There is need for more RCT type research to produce enough high quality evidence for usefulness of nursing CDSS. This will also help to 
derive the much needed standards and guidelines for CDSS in nursing.

b) A critical review of research methods will be useful to judge the appropriateness of research methods used in nursing CDSS research and derive guidelines on this issue.

c) There should be more work to identify contextualised barriers for developing and implementing nursing CDSS. d) If some areas, where nursing CDSS is useful, have been lift out so far, more attention needs to be paid to them in the coming years.

One aspect which has not been researched, but very important, is the relationship or conflict between medicine CDSS and nursing CDSS. If they are not compatible with each other, conflicts between doctors and nurses on decisions are possible.

\section{References:}

Agharezaei, Z., Bahaadinbeigy, K., Tofighi, S., Agharezaei, L., \& Nemati, A. (2014). Attitude of Iranian physicians and nurses toward a clinical decision support system for pulmonary embolism and deep vein thrombosis. Computer methods and programs in biomedicine, 115(2), 95-101. doi:10.1016/j.cmpb.2014.03.007

AHRQ. (2019, June). Clinical Decision Support. Retrieved January 7, 2020, from Agency of Healthcare Research and Quality: https://www.ahrq.gov/cpi/about/otherwebsites/clinicaldecision-support/index.html

Anderson, J. A., \& Willson, P. (2008). Clinical decision support systems in nursing: synthesis of the science for evidence-based practice. Computers, informatics, nursing, 26(3), 151-158. doi:10.1097/01.NCN.0000304783.72811.8e

Arnaert, A., Ponzoni, N., Liebert, J., \& Debe, Z. (2017). Transformative Technology: What Accounts for the Limited Use of Clinical Decision Support Systems in Nursing Practice When Compared to Medicine? In A. Shachak, E. M. Borycki, \& S. P. Reis (Eds.), Health Professionals' Education in the Age of Clinical Information Systems, Mobile Computing and Social Networks (pp. 131-145). Academic Press. doi:10.1016/B978-0-12-805362-1.00007-3

Borum, C. (2018). Barriers for hospital-based nurse practitioners utilizing clinical decision support systems: a systematic review. Computers, Informatics, Nursing, 36(4), 177-182. doi:10.1097/CIN.0000000000000413

Bresnick, J. (2017, December 12). Understanding the Basics of Clinical Decision Support Systems. Retrieved January 7, 2020, from Health IT Analytics: https://healthitanalytics.com/features/understanding-the-basics-of-clinical-decision-supportsystems

CDC. (2018, March 22). Implementing Clinical Decision Support Systems. Retrieved January 7, 2020, from Centre for Disease Control and Prevention: https://www.cdc.gov/dhdsp/pubs/guides/best-practices/clinical-decision-support.htm

Choi, M., Choi, R., Bae, Y.-R., \& Lee, S.-M. (2011). Clinical decision support systems for patient safety: A focus group needs assessment with Korean ICU nurses. Computers, Informatics, Nursing, 29(11), 671-678. doi:10.1097/NCN.0b013e3182148c18

Dowding, D., Mitchell, N., Randell, R., Foster, R., Lattimer, V., \& Thompson, C. (2009). Nurses' use of computerised clinical decision support systems: a case site analysis. Journal of clinical nursing, 18(8), 1159-1167. doi:10.1111/j.1365-2702.2008.02607.x 
Dowding, D., Randell, R., Mitchell, N., Foster, R., Thompson, C., Lattimer, V., \& Cullum, N. (2009). Experience and nurses use of computerised decision support systems. Connecting Health and Humans (Proceedings of NI2009), 146(Studie), 506-510. Retrieved January 8, 2020, from http://eprints. whiterose.ac.uk/43012/6/dowdingD1.pdf

Dunn Lopez, K., Gephart, S. M., Raszewski, R., Sousa, V., Shehorn, L. E., \& Abraham, J. (2017). Integrative review of clinical decision support for registered nurses in acute care settings. Journal of the American Medical Informatics Association, 24(2), 441-450. doi:10.1093/jamia/ocw084

Fossum, M., Alexander, G. L., Ehnfors, M., \& Ehrenberg, A. (2011). Effects of a computerized decision support system on pressure ulcers and malnutrition in nursing homes for the elderly. International journal of medical informatics, 80(9), 607-617. doi:10.1016/j.ijmedinf.2011.06.009

Geurts, D., de Vos-Kerkhof, E., Polinder, S., Steyerberg, E., van der Lei, J., Moll, H., \& Oostenbrink, R. (2017). Implementation of clinical decision support in young children with acute gastroenteritis: a randomized controlled trial at the emergency department. European journal of pediatrics, 176(2), 173-181. doi:10.1007/s00431-016-2819-2

Glasofer, A. (2016). Driving Patient Outcomes through Clinical Decision Support Systems and Nurse-Directed Protocols. 43rd Biennial Convention 2015 Theme: Serve Locally, Transform Regionally, Lead Globally.`, CONV15G07, Las Vegas, Nevada, USA, (p. 28 ppt). Retrieved January 9, 2020, from https://sigma.nursingrepository.org/bitstream/handle/ 10755/603183/1_Glasofer_A_p74654_1.pdf?sequence=1\&isAllowed=y

Gold, D., Hicks, J., Macheska, J., Mason, P., \& McLaughlin, P. (2018). Clinical Decision Support for Emergency Department Nursing Discharge Pain Reassessment. On-Line Journal of Nursing Informatics, 22(3). Retrieved January 9, 2020, from https://search.proquest.com/openview/cc5fded8716865075e8c258bc1957e29/1?pqorigsite $=$ gscholar\&cbl $=2034896$

Hao, A. T.-H., Wu, L.-P., Kumar, A., Jian, W.-S., Huang, L.-F., Kao, C.-C., \& Hsu, C.-Y. (2013). Nursing process decision support system for urology ward. International journal of medical informatics, 82(7), 604-612. doi:10.1016/j.ijmedinf.2013.02.006

Hsiao, J.-L., Wu, W.-C., \& Chen, R.-F. (2013). Factors of accepting pain management decision support systems by nurse anesthetists. BMC medical informatics and decision making, 13(1), 16. Retrieved January 8, 2020, from https://bmcmedinformdecismak.biomedcentral.com/ articles/10.1186/1472-6947-13-16

Jaspers, M. W., Smeulers, M., Vermeulen, H., \& Peute, L. W. (2011). Effects of clinical decision-support systems on practitioner performance and patient outcomes: a synthesis of high-quality systematic review findings. Journal of the American Medical Informatics Association, 18(3), 327-334. doi:10.1136/amiajnl-2011-000094

Johansson-Pajala, R.-M., Gustafsson, L.-K., Blomgren, K. J., Fastbom, J., \& Martin, L. (2017). Nurses' use of computerised decision support systems affects drug monitoring in nursing homes. Journal of nursing management, 25(1), 56-64. doi:10.1111/jonm.12430

Keenan, G. M., Lopez, K. D., Yao, Y., Sousa, V. E., Stifter, J., Febretti, A., . . Wilkie, D. J. (2017). Toward Meaningful Care Plan Clinical Decision Support: Feasibility and Effects of a $\begin{array}{llll}\text { Simulated Pilot Study. } & \text { Nursing research, 66(5), 388-398. }\end{array}$ doi:10.1097/NNR.0000000000000234 
Kim, H., Kim, I., \& Chae, Y. (2006). Design of a nursing clinical decision support system applying nursing diagnosis and nursing evaluation model based data mining. Studies in health technology and informatics, 122(January), 784-785. Retrieved January 8, 2020, from https://europepmc.org/article/med/17102379

Koskela, T., Sandström, S., Mäkinen, J., \& Liira, H. (2015). User perspectives on an electronic decision-support tool performing comprehensive medication reviews-a focus group study with physicians and nurses. BMC medical informatics and decision making, 16(1), 6. Retrieved January $8, \quad 2020$, from https://bmcmedinformdecismak.biomedcentral.com/ articles/10.1186/s12911-016-0245-Z

Lee, S. (2013). Features of computerized clinical decision support systems supportive of nursing practice: a literature review. CIN: Computers, Informatics, Nursing, 31(10), 477-495. doi:10.1097/01.NCN.0000432127.99644.25

Lyerla, F. (2008). Design and implementation of a nursing clinical decision support system to promote guideline adherence. CIN: Computers, Informatics, Nursing, 26(4), 227-233. doi:10.1097/01.NCN.0000304800.93003.b4

Lyerla, F., LeRouge, C., Cooke, D. A., Turpin, D., \& Wilson, L. (2010). A nursing clinical decision support system and potential predictors of head-of-bed position for patients receiving mechanical ventilation. American Journal of Critical Care, 19(1), 39-47. doi:10.4037/ajcc2010836

Müller-Staub, M., de Graaf-Waar, H., \& Paans, W. (2016). An internationally consented standard for nursing process-clinical decision support systems in electronic health records. Computers, Informatics, Nursing, 34(11), 493-502. Retrieved January 8, 2020, from http://www.acendio.net/An_Internat_Consented_NP-CDSS_Standard.pdf

ONC. (2019). Clinical Decision Support. Retrieved January 7, 2020, from HealthIT.Gov: https://www.healthit.gov/topic/safety/clinical-decision-support

Petrucci, K., Petrucci, P., Canfield, K., McCormick, K. A., Kjerulff, K., \& Parks, P. (1991). Evaluation of UNIS: urological nursing information systems. Proceedings of the Annual Symposium on Computer Application in Medical Care (pp. 43-47). American Medical Informatics Association. Retrieved January 7, 2020, from https://www.ncbi.nlm.nih.gov/pmc/articles/PMC2247492/pdf/procascamc00004-0068.pdf

Piscotty, R., \& Kalisch, B. (2014). Nurses' use of clinical decision support: a literature review. Computers, Informatics, Nursing, 32(12), 562-568. doi:10.1097/CIN.0000000000000110

Randell, R., \& Dowding, D. (2010). Organisational influences on nurses' use of clinical decision support systems. International journal of medical informatics, 79(6), 412-421. doi:10.1016/j.ijmedinf.2010.02.003

Randell, R., Mitchell, N., Dowding, D., Cullum, N., \& Thompson, C. (2007). Effects of computerized decision support systems on nursing performance and patient outcomes. Journal of Health Services Research and Policy, 12(4), 242-251. doi:10.1258/135581907782101543

Ruland, C. M. (1999). Decision support for patient preference-based care planning: effects on nursing care and patient outcomes. Journal of the American Medical Informatics Association, 6(4), 304-312. doi:10.1136/jamia.1999.0060304

Ruland, C. M. (2002). Handheld technology to improve patient care: evaluating a support system for preference-based care planning at the bedside. Journal of the American Medical Informatics Association, 9(2), 192-201. doi:10.1197/jamia.M0891

Susan Cortez, B. S. (2016). Measuring clinical decision support influence on evidence-based nursing practice. Oncology nursing forum, 43(4), E170. doi:10.1188/16.ONF.E170-E177 
Thompson, C., Aitken, L., Doran, D., \& Dowding, D. (2013). An agenda for clinical decision making and judgement in nursing research and education. International Journal of Nursing Studies, 50(12), 1720-1726. doi:10.1016/j.ijnurstu.2013.05.003

Van de Velde, S., Heselmans, A., Delvaux, N., Brandt, L., Marco-Ruiz, L., Spitaels, D., \& Cloetens, H. e. (2018). A systematic review of trials evaluating success factors of interventions with computerised clinical decision support. Implementation science, 13(1), 114. Retrieved January $8, \quad 2020$, from https://implementationscience.biomedcentral.com/articles/ $10.1186 / \mathrm{s} 13012-018-0790-1$

\section{Mohammad Ayed}

\section{Aloufi}

Bisha University/ KSA,

Saudi Arabia

mohdaloufi@hotmail.com 